\title{
Desproporción prótesis-paciente luego de reemplazo de válvula aórtica y seguimiento a 6 años
}

\author{
Carolina Carrizo ${ }^{1}$, Virginia Figueroa ${ }^{1}$, Gabriela Vilte $^{2}$, Nicolás Egea $^{2}$, Juan González Grima ${ }^{1}$, Brunella Bertazzo ${ }^{1}$, Eduardo Brenna ${ }^{3}$, \\ Marcos Amuchastegui ${ }^{3}$, Alejandro Contreras ${ }^{3}$ \\ 1.Médico residente de Cardiología \\ 2. Médico especialista en cardiología \\ 3. Cardiólogo \\ Hospital Privado Universitario de Córdoba. \\ El estudio fue realizado en Hospital Privado Universitario de Córdoba ubicado en la ciudad de Córdoba. \\ No tuvo apoyo financiero.
}

Introducción: La desproporción prótesis-paciente (DPP) tras cirugía valvular ocurre cuando el área efectiva de orificio protésico (AEO) es fisiológicamente demasiado pequeño en relación con el tamaño del paciente, lo que resulta en gradientes postoperatorios elevados.

Material y métodos: Se realizó un estudio observacional retrospectivo. Se analizaron pacientes con estenosis aórtica sometidos a reemplazo de válvula aórtica durante el año 2010. Se calculó AEO/ASC postoperatorio, si AEO/ASC fue menor de $0,85 \mathrm{~cm} 2 /$ m2 el paciente fue clasificado como desproporción prótesis-paciente. Los eventos clínicos analizados fueron: mortalidad por cualquier causa, recambio valvular, internación por insuficiencia cardiaca y la capacidad funcional al momento de la encuesta. Se realizaron esta- dísticas descriptivas para analizar las características de la población. Las variables categóricas se expresaron en porcentaje y las variables continuas en promedio y desviación estándar. El análisis estadístico se realizó con el software IBM ${ }^{\circledR}$ SPSS ${ }^{\circledR} 19$.

Resultados: Se analizaron 26 pacientes sometidos a reemplazo de válvula aórtica con un seguimiento a 6 años, 13 de ellos presentaron DPP y mayor porcentaje de disnea, internación por insuficiencia cardiaca y muerte por cualquier causa. La combinación de eventos clínicos en este grupo de pacientes fue del 53,9\%. Conclusión: Se observó un mayor número de eventos cardiovasculares en aquellos pacientes que con desproporción prótesis-paciente.

Palabras clave: Desproporción prótesis paciente, estenosis aórtica, reemplazo valvular aórtico.

\section{Correspondencia:}

Dra. Carolina Carrizo

Naciones Unidas 346, Córdoba, Córdoba (5000). Argentina. Teléfono de trabajo:

+54 (0351) 4688220. Celular: +54 9(3549) 464759 .

carolinacarrizo89@gmail.com. 


\section{Patient-prosthesis mismatch in aortic valve replacement: a 6 year follow up}

Background: Prosthesis-patient mismatch (PPM) after aortic valve replacement is related to inferior long-term outcomes. The study aim was to describe the rate of cardiovascular events in patients with or without PPM.

Methods: The study was based on a retrospective analysis of information obtained from the electronic medical record. All patients undergoing aortic valve replacement, with or without revascularization surgery during 2010 were included. The effective orifice area (EOA) of the prothesis was obtained based on prosthetic valve data from echocardiography recommendations, was related to body surface area (BSA). PPM was diagnosed when EOA / BSA was $<0.85 \mathrm{~cm} 2 / \mathrm{m} 2$. Clinical results were evaluated in January 2017 through our electronic medical record data base and a telephone interview. Helsinki criteria for clinical research were respected.

Results: 26 patients were analyzed (20 males) with mean age 64 (SD 11.5) years old. A mechanical pros- thesis was implanted in 10 patients and a biological one in the remaining subjects. 13 patients had DPP (EOA/BSA $0.77 \pm 0.06$ ). At a mean follow up period of 2190 days $44 \%$ were in functional class (NYHA) II-III, $31 \%$ had been re-hospitalized for heart failure and $8 \%$ had died from cardiac causes (overall death rate $31 \%$ ). The combined outcome rate (overall death, hospitalization for heart failure or re-replacement of the valve) was $54 \%$. Among the 13 patients without PPM, $31 \%$ were in functional class II-III, there were no hospitalizations for heart failure and only 4 patients had died from cardiac causes. Combined outcome rate was $23 \%$.

Conclusions: PPM was a marker of poorer clinical results on a long term follow up of patients undergoing aortic valve replacement. Inferential statistical analysis was not performed due to the relatively low number of patients included.

Keywords: Prosthesis-patient mismatch; aortic valve, stenosis; valve replacement, aortic. 


\section{Introducción:}

El problema de la desproporción prótesis-paciente (DPP) tras cirugía valvular fue descrito por primera vez en $1978^{1}$, ocurriendo esto cuando el área efectiva de orificio protésico (AEO) es fisiológicamente demasiado pequeño en relación con el tamaño del paciente, lo que resulta en gradientes postoperatorios anormalmente altos. De alguna manera se puede predecir la ocurrencia de DPP al conocer el tamaño de la válvula a implantar y la superficie corporal del paciente. La magnitud de la DPP se estima según la razón AEO indexada a la superficie corporal del paciente $(\mathrm{ASC})^{2-3}$.

Existen datos encontrados sobre la capacidad de este método para predecir resultados clínicos a largo plazo, luego del reemplazo valvular aórtico ${ }^{4-5}$.

El objetivo del estudio fue describir la tasa de eventos cardiovasculares en pacientes con desproporción prótesis-paciente teórica.

\section{Material y Métodos:}

Se realizó un estudio observacional retrospectivo. Los datos demográficos de los pacientes y los datos sobre la cirugía cardíaca, así como internaciones posteriores fueron adquiridos desde la base de datos de historia clínica electrónica. Se incluyeron en el estudio todos los pacientes con estenosis aórtica severa sintomática que fueron sometidos a reemplazo de válvula aórtica o combinada con cirugía de revascularización miocárdica en esta institución en el período de enero del 2010 a diciembre del 2010.

El AEO de cada válvula empleada se obtuvo a partir de los datos aportados en las recomendaciones ecocardiográficas de prótesis valvulares ${ }^{6}$.

Según la AEO/ASC calculada después de la operación, si $\mathrm{AEO} / \mathrm{ASC}$ fue menor de $0,85 \mathrm{~cm} 2 / \mathrm{m} 2$ el paciente fue clasificado como desproporción prótesis-paciente.

La capacidad funcional se evaluó en enero de 2017 mediante entrevista telefónica utilizando el formulario corto. El médico que realizó la entrevista leyó las preguntas tal como estaban preestablecidas, se les hicieron preguntas para estimar su estado funcional según la clasificación de New York Heart Association (NYHA). También el cuestionario incluyo datos adicionales sobre mortalidad, hospitalizaciones por insuficiencia cardiaca y limitaciones de funciones debidas a otras patologías.

Los eventos clínicos que se tuvieron en cuenta en el seguimiento fueron: muerte por cualquier causa, recambio valvular, internación por insuficiencia cardiaca y capacidad funcional al momento de la encuesta.

Se realizaron estadísticas descriptivas para analizar las ca- racterísticas de la población.

Las variables categóricas se expresaron en porcentaje y las variables continuas en promedio y desviación estándar. El análisis estadístico se realizó con el software IBM® SPSS 119.

\section{Consideraciones éticas}

El estudio se realizó de acuerdo con normativas para estudios observacionales, respetándose los principios de la declaración de Helsinki.

\section{Resultados:}

Un total de 26 pacientes fueron sometidos a reemplazo de válvula aórtica durante el año 2010, (20 hombres, 6 mujeres), de edad media $64 \pm 11,5$ años. Todos ellos tenían estenosis aórtica severa, sometidos a reemplazo de válvula aórtica, 10 prótesis mecánicas $(38,5 \%)$ y 16 válvulas biológicas $(61,5 \%)$. El 34,6\% de las cirugías fueron combinadas con revascularización miocárdica. (Tabla 1).

\begin{tabular}{|ll|}
\hline Tabla 1. Características basales de los pacientes. \\
\hline Características & $\mathrm{n}=26$ \\
\hline Edad -- años & $64 \pm 11,6$ \\
Sexo masculino -- \% & 76,9 \\
\hline Superficie corporal -- m2 & $1,98 \pm 0,18$ \\
Hipertensión arterial -- $\%$ & 73 \\
\hline CRM combinado --\% & 34,6 \\
\hline Prótesis valvulares biológicas --\% & 61,5 \\
\hline Prótesis valvulares mecánicas -- \% & 38,5 \\
\hline Cirugía de revascularización miocárdica (CRM). Área del orificio \\
efectivo (AOE). Área de superficie corporal (ASC).
\end{tabular}

Se calculó en el postoperatorio el AEO / ASC; el período de seguimiento clínico fue de 2.190 días (6 años). Al finalizar se llevó a cabo una entrevista telefónica para recabar datos sobre estado de capacidad funcional, hospitalizaciones por insuficiencia cardíaca en otra institución, limitaciones por otras patologías.

Del total de los pacientes, 13 pacientes cumplieron criterios para desproporción prótesis-paciente con una AEO/ASC media de $0.77 \pm 0.06$. La edad promedio fue de $62,7 \pm 10,3$; mayoría sexo masculino $(76,9 \%)$, con una superficie corporal media de $2,04 \pm 0,17$. Las prótesis valvulares implantadas fueron biológicas en el 53,8\%. Debido a enfermedad coronaria concomitante, se realizó 
cirugía de revascularización combinada en el 23\% de los casos. (Tabla 2).

\begin{tabular}{|c|c|}
\hline \multicolumn{2}{|c|}{$\begin{array}{l}\text { Tabla 2. Características basales de los pacientes con } \\
\text { desproporción prótesis paciente (DPP). }\end{array}$} \\
\hline Características & DPP $n=13$ \\
\hline Edad -- años & $62,7 \pm 10.3$ \\
\hline Sexo masculino --\% & 76,9 \\
\hline Superficie corporal --m2 & $2,04 \pm 0,17$ \\
\hline Hipertensión arterial --\% & 88,9 \\
\hline CRM combinado -- \% & 23 \\
\hline Válvulas biológicas -- \% & 53,8 \\
\hline AOE/ASC -- cm2/m2 & $0,77 \pm 0,06$ \\
\hline
\end{tabular}

En el seguimiento entre los pacientes DPP teórica, el 44 $\%$ presentaron disnea clase funcional II-III de NYHA, internación por insuficiencia cardiaca ocurrió en el 31\%, recambio valvular en el $8 \%$, muerte por cualquier causa en el $31 \%$. La tasa de eventos combinados de: muerte por cualquier causa, internación por insuficiencia cardiaca y recambio valvular fue del $53.9 \%$ (Tabla 3 ).

Tabla 3. Eventos cardiovasculares a 6 años de seguimiento en pacientes con desproporción prótesis paciente (DPP).

$\begin{array}{ll}\text { Eventos } & \text { DPP } n=13 \\ \text { Eventos combinados de muerte por } & \\ \text { cualquier causa, recambio valvular e } & \\ \text { internación por insuficiencia cardíaca. -no. (\%) } & 7(53,9) \\ \text { Recambio valvular - no. (\%) } & 1(8) \\ \text { Internación por Insuficiencia cardíaca --no. (\%) } & 4(31) \\ \text { Capacidad Funcional NYHA II-III - no. (\%) } & 4(44,, 4)\end{array}$

Entre los 13 pacientes restantes que no calificaron para DPP, $4(30,7 \%)$ presentaron disnea de esfuerzo clase funcional II-III de NYHA. No presentaron internaciones por insuficiencia cardíaca, ni cirugía de recambio valvular. Muerte por cualquier causa ocurrió en 3 pacientes, de los cuales ninguno fue de causa cardiovascular. El porcentaje de eventos clínicos combinados (muerte por cualquier causa, internación por insuficiencia cardiaca y recambio valvular) fue del $23,1 \%$ ( 3 pacientes).

\section{Discusión}

Existen varias estrategias preventivas para evitar o minimizar DPP. En nuestro trabajo se aplicó para la definición de desproporción prótesis-paciente el método del AEO proyectada, es decir, una forma de predecir el AEO antes de implantar la válvula ${ }^{7}$. Este método tiene la importancia de ser útil para evitar la DPP, usando prótesis de tamaños más grandes al momento de la cirugía y con mejores perfiles hemodinámicos ${ }^{8}$, aunque no siempre es posible debido a las características anatómicas del paciente.

Estudios clínicos usando este método han demostrado el efecto negativo de la DPP tras reemplazo valvular aórtico sobre la regresión de la masa del ventrículo izquierdo, recuperación de la función sistólica de VI, clase funcional de la NYHA, calidad de vida, durabilidad de la prótesis $^{7,9}$, e incluso una incidencia incrementada de mortalidad y eventos cardiacos posteriores a la desproporción prótesis- paciente ${ }^{10,11}$.

Sin embargo, el impacto del desajuste prótesis-paciente sigue sin resolverse y los resultados controvertidos. En algunos estudios, DPP tuvo un impacto en la sobrevida a largo plazo y muertes cardiovasculares ${ }^{12,13}$, mientras que en otros no hubo diferencia significativa en comparación con aquellos que no presentaban DPP ${ }^{14,15}$. Los estudios son más uniformes con respecto a la capacidad funcional, aunque sin encontrar diferencias en los pacientes de mayor edad ${ }^{16,18}$.

Se ha visto que no siempre un AEO proyectada, se ve reflejada posteriormente en gradientes transvalvulares elevados en el postoperatorio alejado del paciente ${ }^{19,20}$, posiblemente debido a que los gradientes no sólo dependen del área de la prótesis, sino que también de la geometría ventricular, la función sistólica del ventrículo izquierdo, el llenado ventricular y el acoplamiento ventrículo arterial entre otras variables.

En nuestro trabajo en número de eventos cardiovasculares en pacientes con DPP no son despreciables y son más frecuentes que en aquellos sin DPP, aunque este ensayo carece de poder estadístico para demostrarlo debido al tamaño muestral.

Pensamos que este método de predicción tiene importancia en la práctica cotidiana. Es un dato más para tener en cuenta a la hora de la elección valvular y puede influir en la estrategia quirúrgica. 


\section{Referencias}

1. RAHIMTOOLA SH. The problem of valve prosthesis-patient mismatch. Circulation. 1978;58:20-4.

2. DUMESNIL JG, HONOS GN, LEMIEUX M, BEAUCHEMIN $J$. Validation and applications of indexed aortic prosthetic valve areas calculated by Doppler echocardiography. J Am Coll Cardiol. 1990;16:637-43.

3. PIBAROT P, DUMESNIL JG, LEMIEUX M, CARTIER P, MÉTRAS J, DURAND LG. Impact of prosthesis-patient mismatch on hemodynamic and symptomatic status, morbidity and mortality after aortic valve replacement with a bioprosthetic heart valve. J Heart Valve Dis. 1998;7:211-8.

4. BLEIZIFFER S, EICHINGER WB, HETTICH I, GUENZINGER R, RUZICKA D, BAUERNSCHMITT R, et al. Prediction of valve prosthesis-patient mismatch prior to aortic valve replacement: which is the best method? Heart. 2007;93:615-20.

5. PIBAROT P, DUMESNIL JG. Prevention of valve prosthesis--patient mismatch before aortic valve replacement: does it matter and is it feasible? Heart. 2007;93:549-51.

6. LANCELLOTTI P, PIBAROT P, CHAMBERS J, EDVARDSEN T, DELGADO V, DULGHERU R, et al. Recommendations for the imaging assessment of prosthetic heart valves: a report from the European Association of Cardiovascular Imaging endorsed by the Chinese Society of Echocardiography, the Inter-American Society of Echocardiography, and the Brazilian Department of Cardiovascular Imaging. Eur Heart J Cardiovasc Imaging. 2016;17:589-90.

7. PIBAROT P, DUMESNIL JG. Prosthesis-patient mismatch: definition, clinical impact, and prevention. Heart. 2006;92:1022-9.

8. RUBENS FD, RAO RV, CHAN V, BURWASH IG. A Matched-Paired Comparative Analysis of the Hemodynamics of the Trifecta and Perimount Aortic Bioprostheses. J Heart Valve Dis. 2015;24:487-95.

9. FLAMENG W, HERREGODS MC, VERCALSTEREN M, HERIJGERS P, BOGAERTS K, MEURIS B. Prosthesis-patient mismatch predicts structural valve degeneration in bioprosthetic heart valves. Circulation. 2010;121:2123-9.

10. BLAIS C, DUMESNIL JG, BAILLOT R, SIMARD S, DOYLE D, PIBAROT P. Impact of valve prosthesis-patient mismatch on short-term mortality after aortic valve replacement. Circulation. 2003;108:983-8.

11. WALTHER T, RASTAN A, FALK V, LEHMANN S, GARBADE J, FUNKAT AK, et al. Patient prosthesis mismatch affects short- and long-term outcomes after aortic valve replacement. Eur J Cardiothorac Surg. 2006;30:15-9.

12. HONG S, YI G, YOUN YN, LEE S, YOO KJ, CHANG BC. Effect of the prosthesis-patient mismatch on long-term clinical outcomes after isolated aortic valve replacement for aortic stenosis: a prospective observational study. J Thorac Cardiovasc Surg. 2013;146:1098-104

13. RAO V, JAMIESON WR, IVANOV J, ARMSTRONG S, DAVID TE. Prosthesis-patient mismatch affects survival after aortic valve replacement. Circulation. 2000;102:III5-9.

14. HERNANDEZ-VAQUERO D, GARCIA JM, DIAZ R, CALVO D, KHALPEY Z, HERNANDEZ E, et al. Moderate patient-prosthesis mismatch predicts cardiac events and advanced functional class in young and middle-aged patients undergoing surgery due to severe aortic stenosis. J Card Surg. 2014;29:127-33.

15. CONCISTRÈ G, DELL'AQUILA A, PANSINI S, CORSINI B, COSTIGLIOLO T, PICCARDO A, et al. Aortic valve replacement with smaller prostheses in elderly patients: does patient prosthetic mismatch affect outcomes? J Card Surg. 2013;28:341-7.

16. HERNANDEZ-VAQUERO D, CALVO D, GARCIA JM, LOZANO I, MORALES C, NAYA JL, et al. [Influence of patient-prosthesis mismatch in the octogenarian undergoing surgery for aortic valve replacement due to severe stenosis]. Rev Esp Cardiol. 2011;64:774-9.

17. SPORTELLI E, REGESTA T, SALSANO A, GHIONE P, BREGA C, BEZANTE GP, et al. Does patient-prosthesis mismatch after aortic valve replacement affect survival and quality of life in elderly patients? J Cardiovasc Med (Hagerstown). 2016;17:137-43.

18. MOON MR, LAWTON JS, MOAZAMI N, MUNFAKH NA, PASQUE MK, DAMIANO RJ. POINT: Prosthesis-patient mismatch does not affect survival for patients greater than 70 years of age undergoing bioprosthetic aortic valve replacement. J Thorac Cardiovasc Surg. 2009;137:278-83.

19. DEL CORRO I, CONTRERAS A, BRENNA E. ¿Es posible predecir gradientes transvalvulares elevados en la cirugía valvular aórtica? Rev Fed Arg Cardiol. 2013;43:14-7.

20. CASTRO E, CAVAZZI M, CURCIO A, BALTRUCOWICZ C, VENDITTI R, GUIDOBONO P, et al. Mismatch paciente-prótesis aórtica. Comparación alejada por eco-Doppler transtorácico versus el estimado en el preoperatorio. Rev Argent Cardiol. 2008;76:106-11. 